\section{Recent Advances in the Analysis of PCBs and Pesticides in Human Adipose Tissue}

\section{James C. Peterson and Peter Robinson}

\author{
Pacific Toxicology Laboratories \\ 1545 Pontius Avenue \\ Los Angeles, CA 90025
}

\section{Introduction}

Due to their bioaccumulative and persistent nature, determination of $\mathrm{PCB}$ and organochlorine pesticide levels in human adipose tissue can be a useful biological indicator of exposure. The analytical methods previously used are relatively tedious, labor intensive and not readily amenable to automation. We have combined the use of sweep codistillation cleanup [1] and high resolution gas chromatography with automated on-column injection to efficiently process and quantitate levels of these compounds in fat samples.

\section{Methods}

Tissue samples (200 to $500 \mathrm{mg}$ ) are dried with sodium sulfate and extracted three times with petroleum ether. The solvent is totally removed and the 100 to $500 \mathrm{mg}$ of the remaining extracted fat is ready for cleanup by sweep co-distillation (SGE, Australia). The principle of sweep co-distillation is based on the differential volatility of fat and the organochlorine compounds of interest and utilizes a process which is similar to a preparative gas chromatograph. The rendered or extracted fat is injected directly into a heated tube filled with uncoated glass beads. The pesticides and PCBs are carried through the column by nitrogen, leaving behind the fat. The analytes are retained on a silica gel trap at the end of the glass head column and are eluted from the trap in two solvent fractions.

The first fraction is eluted with $7 \mathrm{~mL}$ of hexane and contains PCBs, hexachlorobenzene, mirex and a portion of the heptachlor $(50 \%)$ and $\mathrm{p}, \mathrm{p}^{\prime}-\mathrm{DDE}$ $(30 \%)$. Fraction II is eluted with $8 \mathrm{~mL}$ of $20 \%$ ethyl ether in hexane and contains p,p'-DDT, p,p'-DDD, p,p'-DDE (70\%), trans-nonachlor, oxychlordane, $\quad \gamma$-chlordane, $\alpha$-chlordane, heptachlor $(50 \%)$, heptachlor epoxide, lindane, $\alpha$-BHC, $\beta$-BHC and Dieldrin. The two fractions are reduced to $2 \mathrm{~mL}$ each before $\mathrm{GC}$ analysis. This cleanup technique allows processing of 10 samples simultaneously, requires relatively little solvent and provides very clean extracts with less than $1 \%$ residual fat. The virtually fat free extracts reduce the problem of capillary column fouling when oncolumn injections are made.

The use of high resolution capillary columns for the separation of PCBs and organochlorine pesticides reduces possible interferences and allows quantitation of individual PCB congeners. This is vital for the analysis of human tissue where metabolism significantly changes the pattern of PCB congeners.

Automated on-column injection (Varian Model 3500, Walnut Creek, CA) allows unattended GC operation while providing the benefits of oncolumn injection including superior injection reproducibility which eliminates the need for an internal standard.

PCB congeners give different electron capture responses, generally increasing with the number of chlorines. Even isomers with the same number of chlorines often have widely varying response factors. Therefore, quantitation based on the assumption of equal response for all congeners in a particular Aroclor mixture will produce inaccurate results, especially in adipose tissue, where metabolism significantly changes the pattern of PCB congeners in a mixture.

In order to determine individual congener response factors, mean weight percents for all the major PCB peaks in each Aroclor mixture were determined by first analyzing the Aroclors by GC/ MS (EI). Assuming that each congener responds equally in the GC/MS, the area percents of the PCB peaks will be approximately equal to the mean weight percent for those peaks. These mean weight percents are used to calculate the amounts of individual congeners in Aroclor standards of known concentration, which, in turn, are used to calculate corresponding ECD response factors. A few of these response factors have been satisfactorily validated analyzing available pure $\mathrm{PCB}$ congener standards (Ultra Chemicals, Hope, RI).

A control lipid sample was prepared by spiking rendered pork lard with $1 \mathrm{mg} / \mathrm{kg}$ of Aroclor 1260 and 0.10 to $0.30 \mathrm{mg} / \mathrm{kg}$ of each of the organochlorine pesticides. 


\section{Results and Discussion}

Quality assurance data collected over a 3 -month period and representing 14 separate runs using the spiked lipid control (approximately once a week), indicates that this procedure produces excellent recoveries and precision (table 1). Each analyte, except for $\beta$-BHC (64\%), has greater than a 78\% recovery. PCBs, hexachlorobenzene and $\mathrm{p}, \mathrm{p}^{\prime}$-DDE are recovered at slightly higher than $100 \%$, most likkely due to endogenous contamination in the lard used for spiking. Precision ranged from 8.3 to $13.5 \%$ relative standard deviation confirming the robust qualities of the method. Even p,p'-DDT which is the poorest performed in the sweep co-distillation process has satisfactory recoveries (78\%) and precision $(11.8 \%)$ indicating that $\mathrm{p}, \mathrm{p}^{\prime}$-DDT breakdown is under control. Detection limits are 0.010 to $0.020 \mathrm{mg} / \mathrm{kg}$ for organochlorine pesticides and $0.050 \mathrm{mg} / \mathrm{kg}$ for PCBs.

Table 1. Recoveries and precision of PCEs and organochlorine pesticides in a spiked pork lard control (14 separate runs over a 3-month period).

\begin{tabular}{|c|c|c|}
\hline Analyte & \% Recovery & $\mathrm{CV}(\%)$ \\
\hline PCB (Aroclor 1260) & 112 & 8.3 \\
\hline Hexachlorobenzene & 105 & 9.1 \\
\hline$\alpha-B H C$ & 93 & 13.5 \\
\hline Lindane & 95 & 10.8 \\
\hline$\beta-B H C$ & 64 & 12.5 \\
\hline trans-Nonachlor & 86 & 12.5 \\
\hline Oxychlordane & 87 & 11.1 \\
\hline Heptachlor epoxide & 95 & 10.8 \\
\hline 'r-Chlondane & 93 & 11.5 \\
\hline$\alpha$-Chiordar.e & \& & 11.5 \\
\hline p. $p^{s}-\mathrm{DDE}$ & $: 14$ & 12.5 \\
\hline p.p'-DDT & 78 & $1 \vdots .8$ \\
\hline Dieldrin & 36 & 126 \\
\hline
\end{tabular}

Adipose tissue results from 136 analyzed human samples are presented in table 2 . As evidenced by these results there are several ubiquitous analytes found in human adipose tissue. PCB levels were found in all samples analyzed and most closely resembled Aroclor 1260, with over $80 \%$ of the total congeners containing six or more chlorines. The relatively narrow concentration range of $\mathrm{PCB}$, hexachlorobenzene, heptachlor epoxide, transnonachlor and oxychlordane residues indicates a similar exposure history to these compounds in the population.

On the other hand, Dieldrin, p,p'-DDT and its metabolite $p, p^{\prime}-D D E$ display a greater concentra- tion variation suggesting individual differences in either exposure or metabolism. a-Chlordane, $\gamma$-cinlordane and heptachlor were present in less than $1 \%$ of the individuals tested. However, their metabolites, oxychlordane and heptachlor epoxide, were always present. Similarly, lindane and $\alpha-B H C$ were rarely detected, but a biorefractory contaminant of technical lindane, $\beta$-BHC, was always present.

Table 2. Chlorinated hydrocarbon residues in 136 adipose tissue extracts analyzed

\begin{tabular}{lccc}
\hline Analyte & Mean, mg/kg & $\begin{array}{c}\text { Standard } \\
\text { deviation }\end{array}$ & $\begin{array}{c}\% \text { of samples } \\
\text { containing } \\
\text { residues }^{*}\end{array}$ \\
\hline PCB & 1.03 & 0.57 & 100 \\
Hexachlorobenzene & 0.048 & 0.027 & 100 \\
B-BHC & 0.087 & 0.061 & 100 \\
Heptachlor epoxide & 0.056 & 0.035 & 100 \\
trans-Nonachlor & 0.122 & 0.070 & 96 \\
Oxychlordane & 0.090 & 0.039 & 100 \\
p,p'-DDE & 1.82 & 1.35 & 100 \\
p,p'-DDT & 0.093 & 0.220 & 75 \\
Dieldrin & 0.055 & 0.066 & 98 \\
\hline
\end{tabular}

"Present in less than 1\%: a-chlordane, $\gamma$-chlordane, lindane, a-BHC.

\section{References}

[1] Luke, B. G., Richards, J. C., and Dawes, E. F., J. Assoc. Off. Anal. Chem. 67, 295 (1984).

\section{Trace/Ultratrace Analyses of Unstable Compounds: Investigations on Hydrazobenzene and Azobenzene}

\author{
S. Ahuja, G. Thompson, and J. Smith \\ Development Department \\ Pharmaceuticals Division \\ CIBA-GEIGY Corporation \\ Suffern, NY 10901
}

Trace analysis generally entails determination at parts per million (ppm) or $\mu \mathrm{g} / \mathrm{g}$ level. Analyses performed at trace or lower levels (ultratrace) are difficult to carry out for several reasons. The difficulties relate to obtaining a representative sample, avoiding loss or contamination during sample preparation, finding a suitable method for resolving the component of interest without significant loss, 